\title{
SCHWARZIAN DERIVATIVES AND ZEROS OF SOLUTIONS TO SECOND ORDER LINEAR DIFFERENTIAL EQUATIONS
}

\author{
A. HINKKANEN AND JOHN ROSSI \\ (Communicated by Clifford J. Earle, Jr.)
}

\begin{abstract}
Let $A$ be entire. Suppose that there exists an unbounded quasidisk $D$ such that $A$ is sufficiently small in $D$. We prove that then any nontrivial solution to $y^{\prime \prime}+A y=0$ has at most one zero in $D$. We show that if $A=$ $Q \exp P$ where $P$ and $Q$ are polynomials, one can usually take $D$ to be an angle of opening $\pi / n$ where $n$ is the degree of $P$.
\end{abstract}

\section{INTRODUCTION}

Consider the differential equation

$$
y^{\prime \prime}(z)+A(z) y(z)=0
$$

where $A$ is an entire function in the complex plane $\mathbb{C}$. All the solutions to (1.1) are known to be entire, and it is natural to analyze their zero distribution near infinity. It seems reasonable to suggest that if $A$ is asymptotically very small in an unbounded domain $D$, then solutions to (1.1) should be asymptotically close to solutions of $y^{\prime \prime}=0$ and hence every nontrivial solution to (1.1) ought to have at most finitely many zeros in $D$. Indeed, recent work of Bank [2] and of Hellerstein and Rossi [4] shows this to be the case when

$$
A(z)=Q(z) e^{P(z)}
$$

where $P$ and $Q$ are polynomials.

We state a simplified version of $[4$, Theorem 3, p. 101]. Let $P$ have degree $n$ and leading coefficient $a_{n}$, and set $l=\operatorname{deg} Q-\operatorname{deg} P$. Fix $\nu \in(0,1], B>0$, and define

$$
V_{k}=\left\{z: 0<\arg z+\frac{\arg a_{n}}{n}-\frac{\pi}{2 n}-\frac{2 \pi}{n}-\frac{2 k \pi}{n}<\frac{\pi}{n}\right\} .
$$

(In [4], the term $\pi / 2 n$ in the definition of $V_{k}$ was inadvertently omitted. It changes nothing in the sequel.)

Received by the editors April 24, 1990; presented to the American Mathematical Society at the regional meeting in Fayetteville, Arkansas on March 23, 1990.

1980 Mathematics Subject Classification (1985 Revision). Primary 30D35, 34A20, 30C60.

Research partially supported by the U. S. National Science Foundation. 
Theorem A. Let $A$ be as in (1.2), and let $f$ be a solution of (1.1), not identically zero. Then $f$ has at most finitely many zeros in

$$
W_{k}=V_{k} \cap\left\{z:|z|^{l+2+\nu}\left|e^{P(z)}\right|<B\right\} .
$$

The reader should note that each $V_{k}$ is a maximal angular region in which $A$ is small. Also, $W_{k}$ is asymptotic to $V_{k}$ in the sense that any closed angle with vertex at the origin that is strictly contained in $V_{k}$ is eventually contained in $W_{k}$.

Our goal in this paper is twofold. First, the proof of Theorem A depends heavily on (1.2) and the shape of $W_{k}$. We extend Theorem A to any function $A(z)$ that is sufficiently small in an unbounded quasidisk. Secondly, Theorem A implies that to each $f$ there exists a positive number $R=R(f)$ such that $f$ has at most one zero in $W_{k} \cap\{z:|z|>R\}$. In general, we are able to remove the dependence of $R$ on $f$, and when $A$ has the form (1.2), we describe how to effectively compute $R$.

\section{THE MAIN RESULT}

Let $f$ and $g$ be linearly independent solutions of (1.1) when $A$ is entire, and define $F=f / g$. It is straightforward to show that $F$ is a locally univalent meromorphic function in $\mathbb{C}$ and that the Schwarzian derivative $S_{F}$ of $F$ satisfies

$$
S_{F}(z) \equiv\left(\frac{F^{\prime \prime}}{F^{\prime}}\right)^{\prime}(z)-\frac{1}{2}\left(\frac{F^{\prime \prime}}{F^{\prime}}(z)\right)^{2}=2 A(z) .
$$

To extend Theorem A to more general functions $A(z)$, we need to ensure that $A(z)$ or, equivalently, $S_{F}(z)$, is sufficiently small. To make this notion precise, we need some definitions.

Let $D$ be a hyperbolic domain in $\mathbb{C}$, that is, a domain that has the unit disk as its universal covering surface. Let $h$ be a locally univalent meromorphic function in $D$. We denote the Poincare density of the hyperbolic metric in $D$ by $\eta_{D}(z)$. The Schwarzian norm $\left\|S_{h}\right\|_{D}$ is defined by

$$
\left\|S_{h}\right\|_{D}=\sup \left\{\left|S_{h}(z)\right| \eta_{D}(z)^{-2}: z \in D\right\} .
$$

Recall that a $K$-quasidisk is the image of the unit disk under a $K$-quasiconformal self-map of $\overline{\mathbb{C}}=\mathbb{C} \cup\{\infty\}$. A domain is a quasidisk if it is a $K$-quasidisk for some $K$. The following theorem is due to Ahlfors [1, pp. 299-300] (cf. [8, p. 81]).

Theorem B. Let $D$ be a $K$-quasidisk. There is a positive constant $\varepsilon(K)$ depending only on $K$ such that every locally univalent meromorphic function $h$ in $D$ satisfying $\left\|S_{h}\right\|_{D}<\varepsilon(K)$ is univalent in $D$.

For a hyperbolic domain $D$, define the inner radius of univalence $\sigma_{I}(D)$ by

$$
\sigma_{I}(D)=\sup \left\{a \geq 0 \text { : if }\left\|S_{h}\right\|_{D} \leq a, \text { then } h \text { is univalent in } D\right\} .
$$


By Theorem B,

$$
\sigma_{I}(D)>0
$$

whenever $D$ is a quasidisk. If $D$ is simply connected, then by a result of Gehring [3] (cf. [8, p. 86]), (2.2) holds only if $D$ is a quasidisk. We remark that if $D$ is a disk or a half plane, a classical result of Nehari [9] shows that $\sigma_{I}(D)=2$.

We may now state our result.

Theorem 1. If $D$ is a hyperbolic plane domain, if $A(z)$ is entire, and if

$$
\sup \left\{|A(z)| \eta_{D}(z)^{-2}: z \in D\right\}<\sigma_{I}(D) / 2,
$$

then every solution to (1.1) that does not vanish identically, has at most one zero in $D$.

The same conclusion holds more generally for $A(z)$ analytic in $D$, provided that (1.1) has two linearly independent single-valued analytic solutions in $D$.

Proof. We may assume that $\sigma_{I}(D)>0$, for otherwise (2.3) is not satisfied by any $A(z)$. Let $f$ be a solution to (1.1) in $D$, not identically zero. By assumption, there is another analytic solution $g$ that is not a constant multiple of $f$. By (2.1), (2.3), Theorem $\mathrm{B}$, and the definition of $\sigma_{I}(D)$, we see that $F=f / g$ is univalent in $D$ and hence has at most one zero there. Since $f$ and $g$ are linearly independent, it follows from the form of (1.1) that their Wronskian $f g^{\prime}-f^{\prime} g$ is a nonzero constant and so $f$ and $g$ have no common zero. Thus, if $f=0$, then also $F=0$, and hence $f$ has at most one zero in $D$. This proves Theorem 1 .

\section{Applications}

3.1. The hypotheses of Theorem 1 involve the Poincare density and the inner radius of univalence, both of which are difficult to compute for general domains, even for quasidisks. We can satisfactorily estimate these quantities as well as $|A(z)|$ when $A$ is of the form (1.2) and the domain $D$ is an angle or close to an angle. From now on we assume that $A$ is given by (1.2) where, without loss of generality,

$$
P(z)=-z^{n}+a_{n-1} z^{n-1}+\cdots+a_{0} .
$$

Let $V_{k}$ be as in (1.3) with $a_{n}=-1$. We prove that every nontrivial solution of (1.1) has at most one zero in a suitable subset of

$$
V_{n-1} \equiv V=\{z:|\arg z|<\pi / 2 n\} \text {. }
$$

Theorem 2. If $A$ and $V$ are as above, and if $n \geq 2$ or $Q$ is constant, then there is an effectively computable nonnegative constant $c$ depending only on $P$ and $Q$ such that any solution to (1.1) that does not vanish identically has at most one zero in

$$
V_{c}=\{z+c: z \in V\} .
$$


A similar result is valid, of course, for any $V_{k}$ instead of $V_{n-1}=V$.

If $n=1$ and $Q$ is not constant, then our method yields the same result only in a smaller and more complicated domain $D$, whose boundary curve is nevertheless asymptotic to $\partial V$ in the sense that any angle strictly inside $V$ is eventually contained in $D$. It will be clear from the proof of Theorem 3 below that it would be technically easier to obtain such a result in a smaller angle strictly contained in $V_{c}$ for a suitable $c$.

Theorem 3. Suppose that $A(z)=Q(z) e^{-z}$ where $Q$ is a nonconstant polynomial, and let $D$ be the domain to the right of the curve

$$
\{\alpha+i y:|y| \leq \beta\} \cup\{x+i y: x \geq \alpha \text { and } x=(d+2) \log r+\gamma\}
$$

where $r^{2}=x^{2}+y^{2}, d=\operatorname{deg} Q, 2 \alpha=(d+2) \log \left(\alpha^{2}+\beta^{2}\right)+2 \gamma$, and $\alpha, \beta$, and $\gamma$ are suitable effectively computable positive constants depending on $Q$ only. Then any nontrivial solution to (1.1) has at most one zero in $D$.

Remarks. (i) Bank [2] and Hellerstein and Rossi [4] also consider functions $A$ of the form

$$
A(z)=\sum_{j=1}^{n} Q_{j}(z) \exp \{j P(z)\}
$$

where $P$ and the $Q_{j}$ are polynomials. Theorems 2 and 3 generalize to this situation in the obvious way.

(ii) If we take

$$
A(z)=\sum_{j=1}^{n} Q_{j}(z) \exp \left\{P_{j}(z)\right\}
$$

where the $P_{j}$ and $Q_{j}$ are polynomials, then it is clear from the proofs that again Theorems 2 and 3 can be extended to cover this case. One replaces $V$ by any angle with a vertex at the origin in which $\operatorname{Re} P_{j}(z)<0$ for $1 \leq j \leq n$ when $|z|$ is large enough. On the other hand, one must now note that for an arbitrary function $A$ of the form (3.4) such angles need not exist, as is shown by the example $A(z)=e^{z}+e^{-z}$.

3.2. Proof of Theorems 2 and 3. Let $V_{c}$ be as in (3.3) with $c>0$. Let $\eta(z)$ denote the Poincaré density of $V_{c}$. Conformal mapping of an angle onto a half plane shows that

$$
\eta(z)=\frac{n|z-c|^{n-1}}{2 \operatorname{Re}(z-c)^{n}} \geq \frac{n}{2|z|}
$$

whenever $c>0$ and $z \in V_{c}$. A result of Lehto and Lehtinen $([7,5]$, cf. [8, p. 123]) shows that

$$
\sigma_{I}\left(V_{c}\right)=2 n^{-2}
$$

Write

$$
Q(z)=b_{d} z^{d}+b_{d-1} z^{d-1}+\cdots+b_{0}
$$


where $d \geq 0$ and $b_{d} \neq 0$ (if $Q \equiv 0$ then Theorem 2 is trivially true). Define

$$
B=\left|a_{n-1}\right|+\left|a_{n-2}\right|+\cdots+\left|a_{0}\right| \text {. }
$$

Clearly there is $c>0$ depending on $P$ and $Q$ only such that

$$
3|A(z)| \eta(z)^{-2} \sigma_{I}\left(V_{c}\right)^{-1} \leq 8\left|b_{d}\right| r^{d+2} \exp \left\{-r^{n} \cos n \theta+2 B r^{n-1}\right\}
$$

for all $z=r e^{i \theta} \in V_{c}$. Thus

$$
\sup \left\{|A(z)| \eta(z)^{-2}: z \in V_{c}\right\} \leq \frac{\sigma_{I}\left(V_{c}\right)}{3}<\frac{\sigma_{I}\left(V_{c}\right)}{2}
$$

provided that

$$
r^{-n}\left\{\log \left(8\left|b_{d}\right|\right)+(d+2) \log r\right\}+2 B r^{-1} \leq \cos n \theta
$$

whenever $r e^{i \theta} \in V_{c}$.

If $n \geq 2$, then (3.7) holds for all large $r$ if $3 B \leq r \cos n \theta$, which is valid for all $r e^{i \theta} \in V_{c}$ if $c$ is large enough, as an elementary estimate shows. Obviously one can obtain an effectively computable $c$ satisfying all the conditions, so that Theorem 2 holds if $n \geq 2$.

If $n=1$ and $Q \equiv b_{0} \neq 0$ is constant, we may assume that $P(z)=-z$, and we return to the equality in (3.5). This shows that

$$
3|A(z)| \eta(z)^{-2} \sigma_{I}\left(V_{c}\right)^{-1}=6\left|b_{0}\right|(x-c)^{2} e^{-x} \leq 24\left|b_{0}\right| e^{-c-2}
$$

for $x>c$ where $z=x+i y$. Thus Theorem 2 holds if we choose

$$
c=\max \left\{0, \log \left(24\left|b_{0}\right| / e^{2}\right)\right\} .
$$

In fact, in this case it would be possible to always choose $c=\log \left(24\left|b_{0}\right| / e^{2}\right)$ and use the definition (3.3) even if $c<0$. This completes the proof of Theorem 2.

If $n=1$ but $Q$ is not constant, we may assume that $P(z)=-z$. We see as above that if $D$ is a domain contained in $V_{c}$ then

$$
\begin{aligned}
3|A(z)| \eta_{D}(z)^{-2} & \leq 3|A(z)| \eta(z)^{-2} \\
& \leq 13\left|b_{d}\right| r^{d}(x-c)^{2} e^{-x} \leq 13\left|b_{d}\right| r^{d+2} e^{-x}
\end{aligned}
$$

if $z=x+i y=r e^{i \theta} \in D$ and $r$ is large enough. Thus if $\sigma>0$, if $D$ is as in Theorem 3 with $\gamma=\log \left(13\left|b_{d}\right| / \sigma\right)$, and if a suitable choice of $\alpha$ (which also determines $\beta$ ) guarantees that $\sigma_{I}(D) \geq \sigma$, then (3.8) implies that

$$
\sup \left\{|A(z)| \eta_{D}(z)^{-2}: z \in D\right\} \leq \sigma / 3<\sigma_{I}(D) / 2 .
$$

Thus Theorem 3 follows from Theorem 1 .

Define $\sigma=2 / 9$. It turns out that if $\beta$ is large enough then for any distinct points $z$ and $w$ in $D$, there is a crescent $E$ with angle $\pi / 3$ with $\{z, w\} \subset$ $E \subset \bar{E} \subset D$. That is, there is a Möbius transformation $M$ such that

$$
E=M(\{z:|\arg z|<\pi / 6\}) .
$$


Thus $\sigma_{I}(E)=\sigma=2 / 9$, by the result of Lehto and Lehtinen [8, p. 123]. The method of proof of Theorem 1 in Lehtinen's paper [6, p. 351] now shows that $\sigma_{I}(D) \geq \sigma$, as required. The calculations required to prove the existence of $E$ for any $z, w \in D$ if $\beta$ is sufficiently large, are routine but tedious, and we omit them. We note that an effectively computable constant $\beta$ emerges in this way. Of course, we also take $\beta$ so large that $|z|=r$ is sufficiently large for all $z \in D$ for (3.8) to hold. This completes the proof of Theorem 3 .

\section{REFERENCES}

1. L. Ahlfors, Quasiconformal reflections, Acta Math. 109 (1963), 291-301.

2. S. Bank, On determining the location of complex zeros of solutions of certain differential equations, Acta Math. Pura Appl. 51 (1988), 67-96.

3. F. W. Gehring, Univalent functions and the Schwarzian derivative, Comment. Math. Helv. 52 (1977), 561-572.

4. S. Hellerstein and J. Rossi, On the distribution of zeros of solutions of second-order differential equations, Complex Variables Theory Appl. 13 (1989), 99-109.

5. M. Lehtinen, On the inner radius of univalency for noncircular domains, Ann. Acad. Sci. Fenn. Ser. A I Math. 5 (1980), 45-47.

6. __ Estimates of the inner radius of univalency of domains bounded by conic sections, Ann. Acad. Sci. Fenn. Ser. A I Math. 10 (1985), 349-353.

7. O. Lehto, Remarks on Nehari's theorem about the Schwarzian derivative and schlicht functions, J. Anal. Math. 36 (1979), 184-190.

8. __ Univalent functions and Teichmüller spaces, Springer, New York, 1987.

9. Z. Nehari, The Schwarzian derivative and schlicht functions, Bull. Amer. Math. Soc. 55 (1949), 545-551.

Department of Mathematics, University of Texas at Austin, Austin, Texas 78712

Current address: Department of Mathematics, University of Illinois at Urbana-Champaign, Urbana, Illinois 61801

Department of Mathematics, Virginia Polytechnic Institute and State University, BLACKSBURG, VIRGINIA 24061-4097 